\title{
Diagnostic and clinical experience of patients with pantothenate kinase- associated neurodegeneration
}

\author{
Randall D. Marshall ${ }^{1}$, Abigail Collins ${ }^{2}$, Maria L. Escolar ${ }^{3}$, H. A. Jinnah ${ }^{4}$, Thomas Klopstock ${ }^{5,6,7}$, Michael C. Kruer ${ }^{8}$, \\ Aleksandar Videnovic ${ }^{9}$, Amy Robichaux-Viehoever ${ }^{10}$, Colleen Burns ${ }^{11^{*}}$, Laura L. Swett ${ }^{12}$, Dennis A. Revicki ${ }^{12}$, \\ Randall H. Bender ${ }^{12}$ and William R. Lenderking ${ }^{12}$
}

\begin{abstract}
Background: Pantothenate kinase-associated neurodegeneration (PKAN) is an autosomal recessive neurodegenerative disorder with brain iron accumulation (NBIA).

Objectives: To assess PKAN diagnostic pathway, history, and burden across the spectrum of PKAN severity from patient and/or caregiver perspectives.

Methods: Caregivers of patients $(n=37)$ and patients themselves $(n=2)$ were interviewed in a validation study of the PKAN-Activities of Daily Living (ADL) scale. The current study used quartiles of the PKAN-ADL total score to divide patients by severity of impairment (Lowest, Second Lowest, Third Lowest, Highest). Diagnostic and treatment history, healthcare utilization, disease burden, and caregiver experience were compared between groups.

Results: The analyses included data from 39 patients. Mean age at PKAN symptom onset $(P=0.0007)$, initial MRI $(P=0.0150)$, and genetic testing $(P=0.0016)$ generally decreased across the PKAN severity spectrum. The mean duration of illness did not differ among PKAN severity groups (range, 9.7-15.2 years; $P=0.3029$ ). First MRI led to diagnosis in 56.4\% of patients (range, 30.0-90.0\%). A mean (SD) of 13.0 (13.1) medical and 55.2 (78.5) therapy visits (eg, physical, speech) occurred in the past year. More patients in the higher PKAN severity groups experienced multiple current functional losses and/or earlier onset of problems ( $P$-values $<0.0500)$. Over half $(56.8 \%)$ of caregivers experienced a change in employment because of caregiving. The percentage of patients requiring fulltime caregiving increased across the PKAN severity spectrum (range, 11.1-100\%; $P=0.0021$ ).

Conclusions: PKAN diagnosis was often delayed, most probably due to low awareness. Considerable burden of functional impairment and high healthcare utilization were found across the PKAN severity spectrum.
\end{abstract}

Keywords: PKAN, Burden of illness, Healthcare utilization, PKAN-ADL scale, Caregiver

\section{Introduction}

Pantothenate kinase-associated neurodegeneration (PKAN) is an autosomal recessive disorder due to mutations in the PANK2 gene, with an estimated prevalence of one to two per million persons worldwide, and a highly variable phenotype [1-7]. PKAN is the most common disorder within the group of neurodegeneration with brain iron accumulation (NBIA) disorders [8]. Motor manifestations of PKAN

\footnotetext{
*Correspondence: colleen.burns@retrophin.com

${ }^{11}$ Retrophin, Inc., 3721 Valley Centre Drive, Suite 200, San Diego, CA 92130, USA

Full list of author information is available at the end of the article
}

include dystonia, chorea, pyramidal signs, parkinsonism, spasticity, dysarthria/anarthria, and dysphagia. Cognitive impairment, psychiatric features, oculomotor deficits, and retinopathy may also be present. The genotype/phenotype association is not well understood, and key features such as rate of progression, age of onset, and signs and symptoms are highly variable, even among siblings and individuals with identical mutations [9].

Because of its rarity, current knowledge about PKAN is based on case reports, case series, and personal observations presented by clinicians from major medical centers. This study was conducted to capture key features

(c) The Author(s). 2019 Open Access This article is distributed under the terms of the Creative Commons Attribution 4.0 International License (http://creativecommons.org/licenses/by/4.0/), which permits unrestricted use, distribution, and 
of the disease history and its progression from the patient and/or caregiver's perspective, in a research sample of 39 patients and their primary caregivers. This relatively large patient sample for a PKAN study provides a good overview of the natural disease history of patients with PKAN, including presenting symptoms, methods of diagnosis, clinical history, and current clinical status. Data were collected in the context of a larger study aiming to develop the first PKAN-specific clinical outcomes assessment scale, the PKAN-Activities of Daily Living (PKAN-ADL) Scale [10].

\section{Methods}

The evaluation of the PKAN-ADL was conducted in accordance with the Declaration of Helsinki, local independent ethics committee/institutional review board requirements, and good clinical practice guidelines. The study protocol received approval by Ethical and Independent Review Services, a central IRB. Written informed consent or assent was obtained from all patients and caregivers.

Study participants were recruited via the NBIA Disorders Association (NBIA-DA), clinicians treating PKAN patients, family networks, and social media. Recruitment continued until the pre-specified study enrollment target was met $(n=40)$. The full inclusion and exclusion criteria for caregivers and patients are previously published [10]. Briefly, the study recruited caregivers $\geq 18$ years old of patients with a genetically confirmed diagnosis of PKAN who were at least 6 years old. Patients who were interviewed had to be at least 16 years old and able to speak clearly enough over the telephone to be interviewed by a stranger. Because difficulty with speech is common in patients with PKAN, verbal interviews are often difficult or impossible. For this reason, we recruited patients with PKAN and their primary caregivers. We interviewed the primary caregiver of a patient with PKAN rather than the patient when PKAN symptoms precluded the possibility of an interview, or if the patient was younger than 16 years old. Patients or caregivers with any clinically relevant physical or mental conditions that would interfere with study participation were excluded.

The measurement evaluation study of the PKAN-ADL consisted of semi-structured interviews via telephone with caregivers and patients. The interview contained a structured evaluation of the PKAN-ADL in addition to an open-ended section about PKAN-related medical history and symptoms (Additional file 1: Table S1). The interviewer administered a patient and/or caregiver sociodemographic information form, a disease-specific history form, and a series of questionnaires to the caregiver and/or patient as appropriate. The interviewer recorded patient and caregiver responses and the audio of the interviews was recorded. Data collection interviews lasted $90-120 \mathrm{~min}$. For the follow-up interview, participants completed the 12-item PKAN-ADL, requiring approximately $20 \mathrm{~min}$, roughly 2 weeks following the initial interview.

\section{Study measures \\ Sociodemographic questionnaires}

The sociodemographic questionnaire for the caregivers and patients included items on age, sex, race/ethnicity, and employment status.

\section{PKAN-specific medical history form}

The PKAN-specific medical history form collected clinical information about the patient's experience with PKAN, including age of onset, duration of illness, method of diagnosis (MRI versus genetic or clinical), standardized questions on milestones of disease progression, and treatments received for PKAN.

\section{PKAN-ADL}

The PKAN-ADL assesses 12 domains of activities of daily living: speech; drooling; swallowing; writing; eating tasks; dressing; personal hygiene tasks; turning or changing position in bed; sitting; falling; walking; and discomfort or pain [10]. Higher scores indicate higher levels of severity of impairment. The five-point scale response options for the PKAN-ADL range from 0 (indicating no problems) to 4 (indicating inability to perform the activity). Quartiles of the scale total summary score were used to define four severity of impairment groups across the spectrum of PKAN severity: Lowest (score < $15)$, Second Lowest $(\geq 15$ to $<25)$, Third Lowest $(\geq 25$ to $<38)$, and Highest $(\geq 38)$.

\section{Other study measures}

Several validated questionnaires were administered within the measurement study to evaluate convergent and divergent validity, as previously published [10]. The measures included selected Quality of Life in Neurological Disorders measures [11], selected attributes of the Health Utilities Index Mark 3 [12], and the Stroke Aphasic Depression Questionnaire [13].

\section{Data analysis}

The data analyses explored descriptive statistics $(\mathrm{N}$, mean, SD, median, minimum, maximum, and floor and ceiling effects) of the PKAN-specific medical history form and the sociodemographic forms for the patients and caregivers. To identify patient groups across the spectrum of PKAN disease severity, since there is no established and validated measure of disease severity or severity-based disease classification to date, we used quartiles of the PKAN-ADL total score to define four 
severity of impairment groups, as described above. Severity of impairment group differences were tested using $\mathrm{F}$ tests and chi-square tests.

\section{Qualitative data analysis}

Qualitative analyses in the study followed the principles of thematic narrative analysis [14] with the goal of identifying themes associated with patient and caregiver feedback regarding the pathway to diagnosis of PKAN, healthcare utilization, and current symptoms and functioning.

\section{Results}

\section{Sociodemographic characteristics of patients and caregivers}

The sample was composed of 37 primary caregivers of patients with PKAN ( $n=37$ patients) and 2 patients with PKAN, for a total of 39 participants providing data on 39 patients (Table 1). Caregivers and patients resided in the United States (US) $(n=35)$, Canada $(\mathrm{n}=3)$, and Germany $(n=1)$. The median age (range) of the caregivers was 45.8 (24.1 to 67.6) years, caregivers were mostly female $(26 / 37 ; 70.3 \%)$, and caregivers were primarily parents of the PKAN patients (33/37; 89.2\%). Most caregivers were related to the patient, lived with the patient, and were employed full-time or part-time (Table 1). Patients $(n=39)$ had a median (range) age of 19.1 (6.4 to 42.6$)$ years, and were mostly White (35/39; $89.7 \%)$, male $(24 / 39 ; 61.5 \%)$, and did not live alone (37/ 39; $94.9 \%)$. A large proportion of patients were students (19/39; 48.7\%); others were employed, unemployed, or disabled (Table 1). Using the quartiles on the PKANADL total score to define four PKAN severity of impairment groups, 9 patients were placed in the Lowest group, 10 in the Second Lowest group, 10 in the Third Lowest group, and 10 in the Highest severity of impairment group. There was a trend toward a difference in age at study enrollment across the PKAN disease spectrum $(P=0.0739) \quad$ (Table 1$)$. The percentages of patients living with someone other than parents or siblings were significantly different across PKAN severity of impairment groups $(P=0.0222)$. No other sociodemographic characteristics significantly differed across PKAN severity groups.

\section{PKAN clinical history and pathway to diagnosis Presenting symptoms of PKAN}

The most common first symptoms or functional limitations noted in patients (often not limited to one symptom) were difficulties with walking, speech, and writing, followed by several other less common initial signs or symptoms such as dystonia or emotional and behavioral problems (Table 2). The percentages of patients presenting with difficulty walking were significantly different
$(P=0.0127)$, showing an increase across the spectrum of PKAN severity. The first PKAN symptom (ie, the presenting symptom, which varied among patients) led to the first doctor visit in $56.4 \%(22 / 39)$ of patients, ranging from $50 \%$ of patients in the Second Lowest (5/10) and the Highest (5/10) groups to $70 \%$ of patients in the Third Lowest $(7 / 10)$ group.

\section{Age at onset}

The median age at symptom onset was 7.0 years. Ages at onset were significantly different across PKAN severity groups $(P=0.0007)$, showing a decrease in mean ages across the disease spectrum (Fig. 1). There was a wide range in mean age at onset across the severity of impairment groups, from 7.0 to 20.0 years old (Lowest), 1.0 to 18.0 years old (Second Lowest), 1.0 to 16.0 years old (Third Lowest), and $<1.0$ to 12.0 years old (Highest), consistent with known PKAN heterogeneity (Table 2). Mean (SD; median) years from symptom onset to PKAN diagnosis was not significantly different across PKAN severity groups $($ Lowest $=3.9[4.4 ; 2.0]$, Second Lowest $=$ $2.6[3.1 ; 1.5]$, Third Lowest $=5.8[9.2 ; 1.0]$, Highest $=4.3$ $[2.9 ; 3.5] ; P=0.6381)$.

\section{First MRI and PKAN diagnosis}

Most patients (38/39 [97.4\%]) had an MRI and the first MRI occurred at a mean (SD) age of 10.4 (6.7) years. Ages at first MRI were significantly different across PKAN severity groups $(P=0.0150)$, generally showing a decrease in mean ages across the disease spectrum (Fig. 1). First MRI led to a diagnosis in just over half of the patients (22/39 [56.4\%]), with no significant difference across PKAN severity groups (Table 2).

\section{Genetic testing and PKAN diagnosis}

Genetic testing was obtained by most patients (37/39 [94.9\%]), leading to a PKAN diagnosis on average at age 12.1 (6.9) years. Ages at diagnosis based on genetic testing were significantly different across PKAN severity groups $(P=0.0116)$, generally showing a decrease in mean ages across the disease spectrum (Fig. 1). Mean (SD; median) years from symptom onset to diagnosis based on genetic testing was not significantly different across PKAN severity groups (Lowest $=4.5[4.4 ; 3.5]$, Second Lowest $=3.1[3.1 ; 4.0]$, Third Lowest $=5.9[9.3$; $1.0]$, Highest $=4.3[2.9 ; 3.5] ; P=0.7584)$.

\section{Physician visits to obtain diagnosis}

The patient journey to diagnosis involved a mean (SD; median) of $4.6(3.5 ; 4.0)$ different physician consultations prior to diagnosis, with a range of 1.0 to 15.0 (Table 2). By PKAN severity, the number of physician consultations prior to diagnosis did not significantly differ across groups $(P=0.4213)$, and ranged 
Table 1 Sociodemographic characteristics

\begin{tabular}{|c|c|c|c|c|c|c|c|}
\hline Characteristic & $\begin{array}{l}\text { Caregivers } \\
(N=37)\end{array}$ & $\begin{array}{l}\text { All patients } \\
(N=39)\end{array}$ & $\begin{array}{l}\text { Lowest } \\
(n=9)\end{array}$ & $\begin{array}{l}\text { Second Lowest } \\
(n=10)\end{array}$ & $\begin{array}{l}\text { Third Lowest } \\
(n=10)\end{array}$ & $\begin{array}{l}\text { Highest } \\
(n=10)\end{array}$ & $P$-value \\
\hline Age, y & $n=35 / 37$ & $n=38 / 39$ & & & $n=9 / 10$ & & 0.0739 \\
\hline Mean (SD) & $46.8(12.5)$ & $20.2(8.4)$ & $24.7(5.8)$ & $19.1(7.1)$ & $22.5(11.0)$ & $15.4(6.9)$ & \\
\hline Median & 45.8 & 19.1 & 22.8 & 19.1 & 23.1 & 14.2 & \\
\hline Range (min, max) & $(24.1-67.6)$ & $(6.4-42.6)$ & $(17.0-33.2)$ & $(6.4-30.0)$ & $(7.9-42.6)$ & $(7.0-29.8)$ & \\
\hline Gender, n (\%) & & & & & & & 0.4626 \\
\hline Male & $11(29.7 \%)$ & $24(61.5 \%)$ & $4(44.4 \%)$ & $8(80.0 \%)$ & $6(60.0 \%)$ & $6(60.0 \%)$ & \\
\hline Female & $26(70.3 \%)$ & $15(38.5 \%)$ & $5(55.6 \%)$ & $2(20.0 \%)$ & $4(40.0 \%)$ & $4(40.0 \%)$ & \\
\hline \multicolumn{8}{|c|}{ With whom patient lives, n (\%) } \\
\hline Parents/Siblings & NA & $33(84.6 \%)$ & $8(88.9 \%)$ & $9(90.0 \%)$ & $6(60.0 \%)$ & $10(100 \%)$ & 0.0222 \\
\hline Spouse/Partner & NA & $0(0.0 \%)$ & $0(0.0 \%)$ & $0(0.0 \%)$ & $0(0.0 \%)$ & $0(0.0 \%)$ & \\
\hline Other family member & NA & $0(0.0 \%)$ & $0(0.0 \%)$ & $0(0.0 \%)$ & $0(0.0 \%)$ & $0(0.0 \%)$ & \\
\hline Alone & NA & $2(5.1 \%)$ & $1(11.1 \%)$ & 1 (10.0\%) & $0(0.0 \%)$ & 0 (0.0\%) & \\
\hline Other & NA & $4(10.3 \%)$ & $0(0.0 \%)$ & $0(0.0 \%)$ & $4(40.0 \%)$ & $0(0.0 \%)$ & \\
\hline
\end{tabular}

Relationship to the patient, $\mathrm{n}(\%)$

$\begin{array}{lll}\text { Parent } & 33(89.2 \%) & \text { NA } \\ \text { Grandparent } & 0(0.0 \%) & \text { NA } \\ \text { Other relative } & 1(2.7 \%) & \text { NA } \\ \text { Professional caregiver } & 1(2.7 \%) & \text { NA } \\ \text { Other } & 2(5.4 \%) & \text { NA }\end{array}$

Caregiver lives with patient, n (\%)

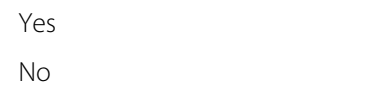

Numbers of hours per week spent with patient

Mean (SD)
Median
Range (min, max)

Ethnic background, $\mathrm{n}$ (\%)

Hispanic or Latino

Not Hispanic or Latino

Racial background, n (\%)

White

Black or African American

Asian

Native Hawaiian or other Pacific Islander

Employment status, n (\%)

Employed, full-time or part-time

Homemaker

Student

Unemployed

Retired

Disabled

$\begin{array}{ll}31(83.8 \%) & \text { NA } \\ 6(16.2 \%) & \text { NA } \\ n=6 / 37 & \\ 16.0(16.7) & \text { NA } \\ 11.0 & \text { NA } \\ (1.0-36.0) & \text { NA }\end{array}$

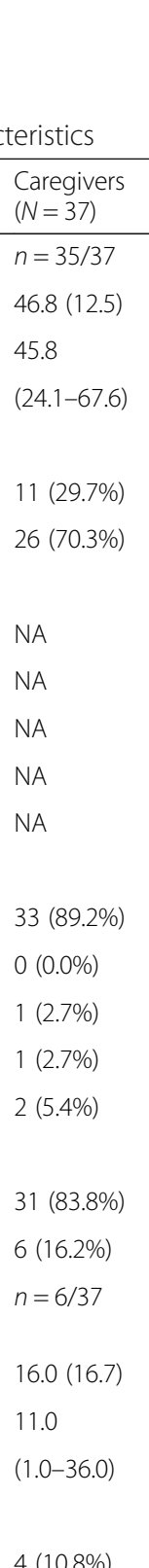

$33(89.2 \%)$$$
6(15.49 \%)
$$$$
2 \text { (22.2\%) }
$$$$
2(20.0 \%)
$$$$
8 \text { (80.0\%) }
$$$$
n=38 / 39
$$$$
33(89.2 \%) \quad 35 \text { (89.7\%) }
$$$$
7(77.8 \%)
$$$$
n=9 / 10
$$$$
8 \text { (88.9\%) }
$$$$
9 \text { (90.0\%) }
$$$$
0(0.0 \%)
$$$$
0(0.0 \%)
$$$$
0(0.0 \%)
$$$$
0(0.0 \%)
$$$$
3(8.1 \%)
$$$$
2(5.1 \%)
$$$$
0(0.0 \%)
$$$$
0(0.0 \%)
$$$$
1(2.7 \%)
$$$$
1(2.6 \%)
$$$$
1(11.1 \%)
$$$$
0(0.0 \%)
$$

\section{$28(75.7 \%)$}

$4(10.3 \%)$

$3(33.3 \%)$

$1(10.0 \%)$

$0(0.0 \%)$

$4(10.8 \%)$

$0(0.0 \%)$

$2(22.2 \%)$

$0(0.0 \%)$

$6(60.0 \%)$

$0(0.0 \%)$

$0(0.0 \%)$

4 (10.3\%)

$1(11.1 \%)$

$0(0.0 \%)$

$4(10.8 \%)$

$0(0.0 \%)$

0 (0.0\%)

1 (2.7\%)

$12(30.8 \%)$

3 (33.3\%)

$3(30.0 \%)$

$\begin{array}{ll}0(0.0 \%) & 2(20.0 \%) \\ 10(100 \%) & 8(80.0 \%) \\ 9(90.0 \%) & 9(90.0 \%) \\ 0(0.0 \%) & 0(0.0 \%) \\ 1(10.0 \%) & 1(10.0 \%) \\ 0(0.0 \%) & 0(0.0 \%)\end{array}$

0.4810

0.5207

0.4198

$\begin{array}{ll}0(0.0 \%) & 0(0.0 \%) \\ 0(0.0 \%) & 0(0.0 \%) \\ 4(40.0 \%) & 7(70.0 \%) \\ 2(20.0 \%) & 1(10.0 \%) \\ 0(0.0 \%) & 0(0.0 \%) \\ 4(40.0 \%) & 2(20.0 \%)\end{array}$


Table 1 Sociodemographic characteristics (Continued)

\begin{tabular}{|c|c|c|c|c|c|c|c|}
\hline Characteristic & $\begin{array}{l}\text { Caregivers } \\
(N=37)\end{array}$ & $\begin{array}{l}\text { All patients } \\
(N=39)\end{array}$ & $\begin{array}{l}\text { Lowest } \\
(n=9)\end{array}$ & $\begin{array}{l}\text { Second Lowest } \\
(n=10)\end{array}$ & $\begin{array}{l}\text { Third Lowest } \\
(n=10)\end{array}$ & $\begin{array}{l}\text { Highest } \\
(n=10)\end{array}$ & $P$-value ${ }^{a}$ \\
\hline Highest educational level, n (\%) & & & & & & & 0.1213 \\
\hline Elementary/primary school & $0(0.0 \%)$ & $14(35.9 \%)$ & $0(0.0 \%)$ & $3(30.0 \%)$ & $5(50.0 \%)$ & $6(60.0 \%)$ & \\
\hline Secondary/high school & $7(18.9 \%)$ & $13(33.3 \%)$ & $2(22.2 \%)$ & $5(50.0 \%)$ & $3(30.0 \%)$ & $3(30.0 \%)$ & \\
\hline Some college & $9(24.3 \%)$ & $9(23.1 \%)$ & $5(55.6 \%)$ & $2(20.0 \%)$ & $1(10.0 \%)$ & $1(10.0 \%)$ & \\
\hline College degree & $7(18.9 \%)$ & $2(5.1 \%)$ & $1(11.1 \%)$ & $0(0.0 \%)$ & $1(10.0 \%)$ & $0(0.0 \%)$ & \\
\hline Some graduate school & $4(10.8 \%)$ & $0(0.0 \%)$ & $0(0.0 \%)$ & $0(0.0 \%)$ & $0(0.0 \%)$ & $0(0.0 \%)$ & \\
\hline Postgraduate degree & $7(18.9 \%)$ & $0(0.0 \%)$ & $0(0.0 \%)$ & $0(0.0 \%)$ & $0(0.0 \%)$ & $0(0.0 \%)$ & \\
\hline Technical or vocational degree & $3(8.1 \%)$ & $0(0.0 \%)$ & $0(0.0 \%)$ & $0(0.0 \%)$ & $0(0.0 \%)$ & $0(0.0 \%)$ & \\
\hline Other & $0(0.0 \%)$ & $1(2.6 \%)$ & $1(11.1 \%)$ & $0(0.0 \%)$ & $0(0.0 \%)$ & $0(0.0 \%)$ & \\
\hline $\begin{array}{l}\text { Educational achievement limited } \\
\text { by PKAN, } n(\%)\end{array}$ & & $n=38 / 39$ & & & $n=9 / 10$ & & 0.1871 \\
\hline Yes & NA & $30(76.9 \%)$ & $5(55.6 \%)$ & $8(80.0 \%)$ & $7(70.0 \%)$ & 10 (100\%) & \\
\hline No & NA & $8(20.5 \%)$ & $4(44.4 \%)$ & 2 (20.0\%) & $2(20.0 \%)$ & $0(0.0 \%)$ & \\
\hline $\begin{array}{l}\text { Change in employment status due } \\
\text { to caregiving, } \mathrm{n}(\%)^{c}\end{array}$ & & & & & & & 0.0128 \\
\hline Yes & $21(56.8 \%)$ & NA & $5(71.4 \%)$ & 9 (90.0\%) & $2(20.0 \%)$ & $5(50.0 \%)$ & \\
\hline No & 16 (43.2\%) & NA & $2(28.6 \%)$ & 1 (10.0\%) & 8 (80.0\%) & $5(50.0 \%)$ & \\
\hline
\end{tabular}

${ }^{a}$ Comparisons between quartile groups based on $\mathrm{F}$ tests for continuous data and chi-square tests for categorical data; ${ }^{\mathrm{b}}$ Responses are not mutually exclusive; ${ }^{\mathrm{C}}$ Employment status change for caregivers is shown by PKAN severity of impairment patient quartile groups

NA, not applicable; PKAN, pantothenate kinase-associated neurodegeneration; SD, standard deviation

from a mean (SD; median) of $3.1(3.2: 2.0)$ in the Second Lowest group to $5.6(3.6 ; 5.5)$ in the Highest group. Eventually, nearly all patients were referred to or initiated contact with specialist centers $(37 / 39$, 94.9\%). One caregiver reported consulting 3 pediatricians, 4 neurologists, and a geneticist outside of the community, prior to obtaining a diagnosis, and driving $3-5 \mathrm{~h}$ round trip per doctor visit. She reported that over a span of 7 years of appointments, the doctors "knew something was wrong" with her child, but were unable to make the diagnosis.

\section{Healthcare utilization Medical care}

The percentages of patients who received various types of treatments (medication, vitamins or over-thecounter treatments, injections, therapies such as physical, speech, or occupational) were similar across PKAN severity groups (Table 3 ). The percentages of patients with surgery other than deep brain stimulation device placement were significantly different across PKAN severity groups $(P=0.0006)$, being more common in the Highest severity group. Across all PKAN patients, a mean (SD; median) of 13.0 (13.1; 8.0) medical visits and $55.2(78.5 ; 22.5)$ therapy visits occurred in the past year. Number of medical or therapy visits did not significantly differ across PKAN severity groups $(P$-values $>0.2187)$ (Table 3$)$.

\section{Caregiving}

Over half of the patients $(21 / 39,53.8 \%)$ required a fulltime caregiver (Table 3). The percentages of patients who required full-time caregiving were significantly different across PKAN severity groups $(P=0.0021)$, showing an increase across the disease spectrum. Median age at onset requiring full-time caregiving ranged from 8.5 years old (Highest group) to 19.0 years old (Lowest group) $(P=0.2379)$, and median age at onset requiring part-time caregiving ranged from 7.0 years old (Highest group) to 19.0 years old (Second Lowest group) $(P=0.0975)$. Reasons given for the need for a full-time caregiver included progression of dementia, unable to be left alone due to both developmental issues and problems with falling, and loss of mobility. Some parents reported sharing caregiving duties with a professional caregiver. Examples of parental caregiving duties included assistance with transportation, aiding patient when patient's hand "tires out" or hand contractions interfere with eating/dressing, assistance with personal hygiene and medications, and monitoring to prevent the patient from wandering off.

Over half $(21 / 37,56.8 \%)$ of caregivers experienced a change in employment status because of caregiving. The percentages of caregivers who experienced a change in employment status were significantly different across PKAN severity groups $(P=0.0128)$, ranging from $20.0 \%$ (2/10, Third Lowest group) to $90.0 \%(9 / 10$, Second 
Table 2 PKAN clinical history and pathway to diagnosis

\begin{tabular}{|c|c|c|c|c|c|c|}
\hline Characteristic & $\begin{array}{l}\text { All patients } \\
(N=39)\end{array}$ & $\begin{array}{l}\text { Lowest } \\
(n=9)\end{array}$ & $\begin{array}{l}\text { Second Lowest } \\
(n=10)\end{array}$ & $\begin{array}{l}\text { Third Lowest } \\
(n=10)\end{array}$ & $\begin{array}{l}\text { Highest } \\
(n=10)\end{array}$ & $P$-value ${ }^{a}$ \\
\hline \multicolumn{7}{|l|}{ Presenting symptoms of PKAN, $n(\%)^{b}$} \\
\hline Walking difficulty & $27(69.2 \%)$ & $3(33.3 \%)$ & $6(60.0 \%)$ & $8(80.0 \%)$ & $10(100 \%)$ & 0.0127 \\
\hline Speech & $12(30.8 \%)$ & $1(11.1 \%)$ & $5(50.0 \%)$ & $2(20.0 \%)$ & $4(40.0 \%)$ & 0.2296 \\
\hline Swallowing & $2(5.1 \%)$ & $1(11.1 \%)$ & $1(10.0 \%)$ & $0(0.0 \%)$ & $0(0.0 \%)$ & 0.5259 \\
\hline Writing & $9(23.1 \%)$ & $4(44.4 \%)$ & $3(30.0 \%)$ & $0(0.0 \%)$ & $2(20.0 \%)$ & 0.1306 \\
\hline Emotional/behavioral problems & $6(15.4 \%)$ & $3(33.3 \%)$ & $1(10.0 \%)$ & $1(10.0 \%)$ & $1(10.0 \%)$ & 0.4080 \\
\hline Other problems & 19 (48.7\%) & $4(44.4 \%)$ & $6(60.0 \%)$ & $6(60.0 \%)$ & $3(30.0 \%)$ & 0.4776 \\
\hline Dystonia at PKAN presentation, $\mathrm{n}(\%)^{\mathrm{b}}$ & $6(15.4 \%)$ & $2(22.2 \%)$ & $2(20.0 \%)$ & $1(10.0 \%)$ & $1(10.0 \%)$ & 0.8176 \\
\hline Dystonia: mouth/tongue & $4(10.3 \%)$ & $2(22.2 \%)$ & $1(10.0 \%)$ & $0(0.0 \%)$ & $1(10.0 \%)$ & \\
\hline Dystonia: neck & $1(2.6 \%)$ & $0(0.0 \%)$ & $0(0.0 \%)$ & $0(0.0 \%)$ & $1(10.0 \%)$ & \\
\hline Dystonia: hand & $6(15.4 \%)$ & $2(22.2 \%)$ & $2(20.0 \%)$ & $1(10.0 \%)$ & $1(10.0 \%)$ & \\
\hline Dystonia: foot & $2(5.1 \%)$ & $1(11.1 \%)$ & $0(0.0 \%)$ & $0(0.0 \%)$ & $1(10.0 \%)$ & \\
\hline Dystonia: back/trunk & $3(7.7 \%)$ & $1(11.1 \%)$ & $1(10.0 \%)$ & $0(0.0 \%)$ & $1(10.0 \%)$ & \\
\hline Dystonia: other & $2(5.1 \%)$ & $1(11.1 \%)$ & $0(0.0 \%)$ & $0(0.0 \%)$ & $1(10.0 \%)$ & \\
\hline Age at onset, y & $n=38 / 39$ & & & $n=9 / 10$ & & 0.0007 \\
\hline Mean (SD) & $8.0(5.8)$ & $12.7(4.3)$ & $9.4(5.5)$ & $7.3(5.2)$ & $2.9(3.8)$ & \\
\hline Median & 7.0 & 14.0 & 10.0 & 7.0 & 1.0 & \\
\hline Range (min, max) & $(<1.0-20.0)$ & $(7.0-20.0)$ & $(1.0-18.0)$ & $(1.0-16.0)$ & $(<1.0-12.0)$ & \\
\hline Problem leading to first doctor visit, n (\%) & $n=36 / 39$ & $n=8 / 9$ & $n=9 / 10$ & $n=9 / 10$ & & \\
\hline Same as "first symptom" & $22(56.4 \%)$ & $5(55.6 \%)$ & $5(50.0 \%)$ & $7(70.0 \%)$ & $5(50.0 \%)$ & \\
\hline Other & $14(35.9 \%)$ & $3(33.3 \%)$ & $4(40.0 \%)$ & $2(20.0 \%)$ & $5(50.0 \%)$ & \\
\hline MRI, n (\%) & & & & & & 0.3953 \\
\hline Yes & $38(97.4 \%)$ & $9(100 \%)$ & $10(100 \%)$ & $10(100 \%)$ & $9(90.0 \%)$ & \\
\hline No & $1(2.6 \%)$ & $0(0.0 \%)$ & $0(0.0 \%)$ & $0(0.0 \%)$ & $1(10.0 \%)$ & \\
\hline Age at first MRI, $y$ & $n=36 / 38$ & $n=8 / 9$ & & $n=9 / 10$ & & 0.0150 \\
\hline Mean (SD) & $10.4(6.7)$ & $15.0(6.9)$ & $11.5(5.0)$ & $10.8(7.8)$ & $5.4(3.8)$ & \\
\hline Median & 10.0 & 16.0 & 10.0 & 10.0 & 4.5 & \\
\hline Range (min, max) & $(1.0-26.0)$ & $(5.0-25.0)$ & $(5.0-23.0)$ & $(1.0-26.0)$ & $(1.0-12.0)$ & \\
\hline Did first MRI diagnose PKAN? & $n=38 / 39$ & & & $n=9 / 10$ & & 0.1005 \\
\hline Yes & $22(56.4 \%)$ & $5(55.6 \%)$ & $9(90.0 \%)$ & $5(50.0 \%)$ & $3(30.0 \%)$ & \\
\hline No & $16(41.0 \%)$ & $4(44.4 \%)$ & $1(10.0 \%)$ & $4(40.0 \%)$ & $7(70.0 \%)$ & \\
\hline Genetic testing obtained & & & & & & 0.5259 \\
\hline Yes & 37 (94.9\%) & $8(88.9 \%)$ & $9(90.0 \%)$ & $10(100 \%)$ & $10(100 \%)$ & \\
\hline No & $2(5.1 \%)$ & $1(11.1 \%)$ & $1(10.0 \%)$ & $0(0.0 \%)$ & $0(0.0 \%)$ & \\
\hline Age genetic testing led to PKAN diagnosis & $n=36 / 39$ & $n=8 / 9$ & $n=9 / 10$ & $n=9 / 10$ & & 0.0116 \\
\hline Mean (SD) & $12.1(6.9)$ & $17.4(4.3)$ & $11.9(5.1)$ & $13.2(9.7)$ & $7.2(3.0)$ & \\
\hline Median & 10.5 & 16.0 & 10.0 & 13.0 & 7.0 & \\
\hline Range (min, max) & $(4.0-36.0)$ & $(12.0-26.0)$ & $(5.0-23.0)$ & $(4.0-36.0)$ & $(4.0-12.0)$ & \\
\hline Age MRI diagnosed PKAN if MRI diagnosis & $n=1 / 22$ & $n=1 / 5$ & $n=0 / 9$ & $n=0 / 5$ & $n=0 / 3$ & \\
\hline Mean (SD) & 11.0 & 11.0 & & & & \\
\hline Median & 11.0 & 11.0 & & & & \\
\hline Range (min, max) & $(11.0-11.0)$ & $(11.0-11.0)$ & & & & \\
\hline
\end{tabular}


Table 2 PKAN clinical history and pathway to diagnosis (Continued)

\begin{tabular}{|c|c|c|c|c|c|c|}
\hline Characteristic & $\begin{array}{l}\text { All patients } \\
(N=39)\end{array}$ & $\begin{array}{l}\text { Lowest } \\
(n=9)\end{array}$ & $\begin{array}{l}\text { Second Lowest } \\
(n=10)\end{array}$ & $\begin{array}{l}\text { Third Lowest } \\
(n=10)\end{array}$ & $\begin{array}{l}\text { Highest } \\
(n=10)\end{array}$ & $P$-value ${ }^{a}$ \\
\hline Number of doctors seen prior to PKAN diagnosis & & & & & & 0.4213 \\
\hline Mean (SD) & $4.6(3.5)$ & $5.2(4.7)$ & $3.1(3.2)$ & $4.5(2.5)$ & $5.6(3.6)$ & \\
\hline Median & 4.0 & 3.0 & 2.0 & 4.0 & 5.5 & \\
\hline Range (min, max) & $(1.0-15.0)$ & $(1.0-15.0)$ & $(1.0-10.0)$ & $(1.0-10.0)$ & $(1.0-12.0)$ & \\
\hline \multicolumn{7}{|l|}{ Location of doctors consulted, $\mathrm{n}(\%)^{\mathrm{b}}$} \\
\hline Community & $16(41.0 \%)$ & $5(55.6 \%)$ & $3(30.0 \%)$ & $6(60.0 \%)$ & $2(20.0 \%)$ & \\
\hline Specialist center doctors & $37(94.9 \%)$ & $7(77.8 \%)$ & $10(100 \%)$ & $10(100 \%)$ & $10(100 \%)$ & \\
\hline
\end{tabular}

${ }^{\mathrm{a}}$ Comparisons among quartile groups based on $\mathrm{F}$ tests for continuous data and chi-square tests for categorical data; ${ }^{\mathrm{b}}$ Responses are not mutually exclusive; 'Patient sample sizes are based on the number of patients reporting first MRI led to diagnosis of PKAN

$\mathrm{MRI}$, magnetic resonance imaging; PKAN, pantothenate kinase-associated neurodegeneration, SD, standard deviation

Lowest group). One parent reported that he retired early to provide full-time care.

\section{Current symptoms and functioning}

The PKAN severity of impairment groups did not significantly differ in mean (SD) years since onset of symptoms $\quad($ Lowest $=12.0$ [5.5], Second Lowest $=9.7$ [3.7], Third Lowest $=15.2$ [9.3], Highest $=12.5$ [5.2] years; $P=$ 0.3029 ) or mean $(\mathrm{SD})$ years since diagnosis (Lowest $=8.1$ [4.6], Second Lowest $=7.1$ [4.6], Third Lowest $=9.4$ [5.8], Highest $=8.2[4.9]$ years; $P=0.8071$ ).

\section{Problems with walking}

Most patients experienced problems with walking (26/ $39,66.7 \%)$. The percentages of patients unable to walk without help and unable to walk at all were significantly different across PKAN severity groups ( $P$-values $\leq 0.0001$ ), generally showing an increase across the disease spectrum (Fig. 2a). Age at onset did not significantly differ for unable to walk without help $(P=0.1447)$, ranging from a mean (SD) age of 7.7 (4.2) years (Highest group) to 13.6 (8.6) years (Second Lowest group), or unable to walk at all $(P=0.3878)$, ranging from 10.4 (7.4) years (Highest group) to 18.0 years in the single patient in the Second Lowest group.

\section{Problems with speaking}

Most patients (36/39 [92.3\%]) had trouble with speaking or being understood. There was no significant difference across PKAN severity groups $(P=0.2671)$, ranging from $80.0 \%$ (8/10 Second Lowest group) to $100.0 \%$ (10/10 each in the Third Lowest and Highest groups). The percentages of patients with lost ability to speak at all were significantly different across PKAN severity groups $(P<0.0001)$, being more common in the Highest severity group (Fig. 2b). Ages at onset for problems with speaking were significantly different across PKAN severity groups $(P=0.0065)$; mean ages generally decreased across the disease spectrum (Fig. 2c).

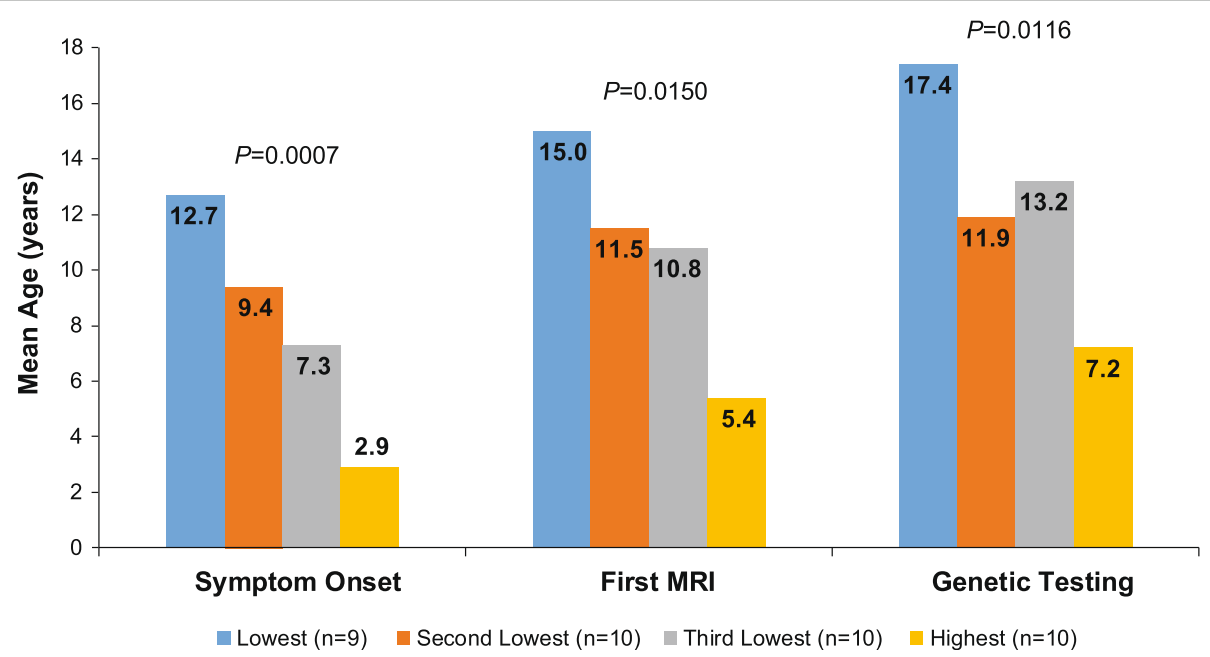

Fig. 1 Mean age at onset of PKAN symptoms, first MRI, and genetic testing. MRI, magnetic resonance imaging; PKAN, pantothenate kinase-associated neurodegeneration 
Table 3 Healthcare utilization

\begin{tabular}{|c|c|c|c|c|c|c|}
\hline & $\begin{array}{l}\text { All patients } \\
N=39\end{array}$ & $\begin{array}{l}\text { Lowest } \\
n=9\end{array}$ & $\begin{array}{l}\text { Second Lowest } \\
n=10\end{array}$ & $\begin{array}{l}\text { Third Lowest } \\
n=10\end{array}$ & $\begin{array}{l}\text { Highest } \\
n=10\end{array}$ & $P$-value \\
\hline \multicolumn{7}{|l|}{ PKAN treatments received } \\
\hline Medication from doctor, n (\%) & $39(100 \%)$ & $9(100 \%)$ & $10(100 \%)$ & $10(100 \%)$ & $10(100 \%)$ & \\
\hline Vitamins or OTC, n (\%) & 35 (89.7\%) & $8(88.9 \%)$ & $7(70.0 \%)$ & $10(100 \%)$ & $10(100 \%)$ & 0.0886 \\
\hline Injections (botulinum toxin, etc), n (\%) & $25(64.1 \%)$ & $4(44.4 \%)$ & $7(70.0 \%)$ & $5(50.0 \%)$ & $9(90.0 \%)$ & 0.1422 \\
\hline $\begin{array}{l}\text { Therapies (physical, speech, occupational, } \\
\text { etc), } n(\%)\end{array}$ & $36(92.3 \%)$ & $7(77.8 \%)$ & $10(100 \%)$ & $9(90.0 \%)$ & $10(100 \%)$ & 0.2198 \\
\hline Surgery, n (\%) & $16(41.0 \%)$ & $2(22.2 \%)$ & $2(20.0 \%)$ & $3(30.0 \%)$ & $9(90.0 \%)$ & 0.0036 \\
\hline DBS device & $9(23.1 \%)$ & $2(22.2 \%)$ & $2(20.0 \%)$ & $2(20.0 \%)$ & $3(30.0 \%)$ & 0.9443 \\
\hline Other surgery & $14(35.9 \%)$ & $2(22.2 \%)$ & $1(10.0 \%)$ & $2(20.0 \%)$ & 9 (90.0\%) & 0.0006 \\
\hline Other treatment, $\mathrm{n}(\%)^{\mathrm{a}}$ & $14(35.9 \%)$ & $1(11.1 \%)$ & $5(50.0 \%)$ & $4(40.0 \%)$ & $4(40.0 \%)$ & 0.3322 \\
\hline \multicolumn{7}{|l|}{ PKAN healthcare utilization past year } \\
\hline Medical visits & & & & & & 0.3446 \\
\hline Mean (SD) & $13.0(13.1)$ & $12.2(16.8)$ & $7.6(5.3)$ & $13.9(15.6)$ & $18.3(11.9)$ & \\
\hline Median & 8.0 & 6.0 & 5.0 & 7.0 & 15.0 & \\
\hline Range (min, max) & $(1.0-56.0)$ & $(2.0-56.0)$ & $(1.0-15.0)$ & $(3.0-50.0)$ & $(1.0-40.0)$ & \\
\hline Therapy visits & $n=38 / 39$ & & & $n=9 / 10$ & & 0.2188 \\
\hline Mean (SD) & $55.2(78.5)$ & $23.1(50.2)$ & $33.7(32.1)$ & $85.1(126.5)$ & $78.7(69.1)$ & \\
\hline Median & 22.5 & 6.0 & 26.5 & 20.0 & 46.0 & \\
\hline Range (min, max) & $(0.0-384.0)$ & $(0.0-156.0)$ & $(0.0-100.0)$ & $(0.0-384.0)$ & $(1.0-208.0)$ & \\
\hline \multicolumn{7}{|l|}{ Caregiving required } \\
\hline Part-time caregiver, n (\%) & $25(64.1 \%)$ & $3(33.3 \%)$ & $6(60.0 \%)$ & $8(80.0 \%)$ & $8(80.0 \%)$ & 0.1656 \\
\hline Age at onset, y & $n=23 / 25$ & $n=1 / 3$ & & & & \\
\hline Mean (SD) & $11.5(7.6)$ & $12.0(\mathrm{NA})$ & $17.7(8.0)$ & $10.8(7.9)$ & $7.6(4.8)$ & 0.0975 \\
\hline Median & 11.0 & 12.0 & 19.0 & 11.0 & 7.0 & \\
\hline Range (min, max) & $(<1.0-28.0)$ & $(12.0-12.0)$ & $(6.0-28.0)$ & $(<1.0-20.0)$ & $(<1.0-14.0)$ & \\
\hline Full-time caregiver, n (\%) & $21(53.8 \%)$ & $1(11.1 \%)$ & $3(30.0 \%)$ & 7 (70.0\%) & $10(100 \%)$ & 0.0021 \\
\hline Age at onset, y & $n=20 / 21$ & & $n=2 / 3$ & & & \\
\hline Mean (SD) & $10.3(6.6)$ & $19.0(\mathrm{NA})$ & $16.5(9.2)$ & $9.7(7.3)$ & $8.5(5.1)$ & 0.2379 \\
\hline Median & 10.0 & 19.0 & 16.5 & 12.0 & 8.5 & \\
\hline Range (min, max) & $(<1.0-23.0)$ & $(19.0-19.0)$ & $(10.0-23.0)$ & $(<1.0-20.0)$ & $(<1.0-17.0)$ & \\
\hline
\end{tabular}

${ }^{a}$ Examples of other treatments for PKAN included leg braces, hippotherapy (horseback riding), and participating in a clinical trial (eg, deferiprone) $D B S$ deep brain stimulation; OTC over the counter; PKAN pantothenate kinase-associated neurodegeneration; SD standard deviation

\section{Problems with eating}

The percentages of patients with eating or choking problems were significantly different across PKAN severity groups $(P=0.0219)$, generally showing an increase across the disease spectrum (Fig. 2a). Age at onset did not significantly differ across PKAN severity groups $(P=0.1064)$, ranging from a mean $(\mathrm{SD})$ age of 9.4 (5.1) years (Highest group) to 18.0 (7.5) years (Lowest group). The need for placement of a feeding tube was significantly different across PKAN severity groups $(P<0.0001)$ and was generally more common in the Highest severity group (Fig. 2b). Age at onset did not significantly differ across PKAN severity groups $(P=0.3626)$, ranging from a mean $(\mathrm{SD})$ age of
10.9 (3.7) years (Highest group) to 14.5 (10.6) years (Third Lowest group), with no patients in the two lowest severity groups reporting requirement of a feeding tube.

\section{Problems with vision and breathing}

Most patients had problems with vision (27/39 [69.2\%]). The percentages of patients with vision problems were significantly different across PKAN severity groups $(P=$ 0.0313), generally showing an increase across the disease spectrum (Fig. 2a). There was a trend toward different ages at onset for problems in vision across PKAN severity groups $(P=0.0789$, Fig. 2 c). One patient each in the 


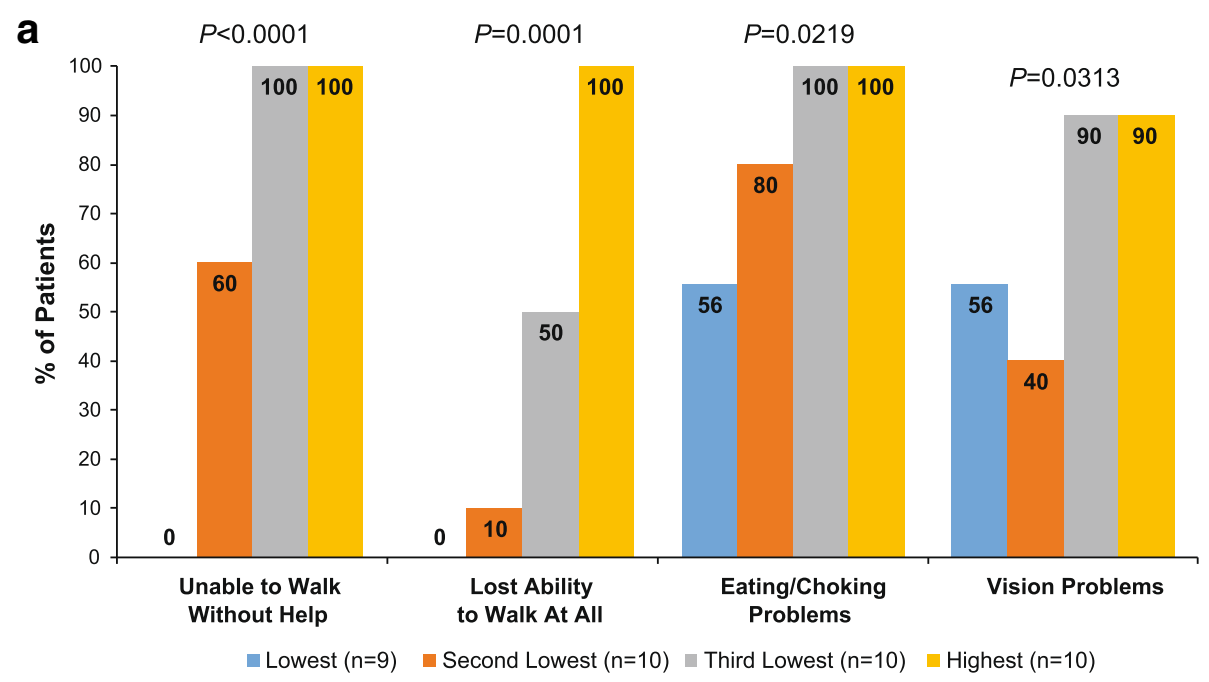

b

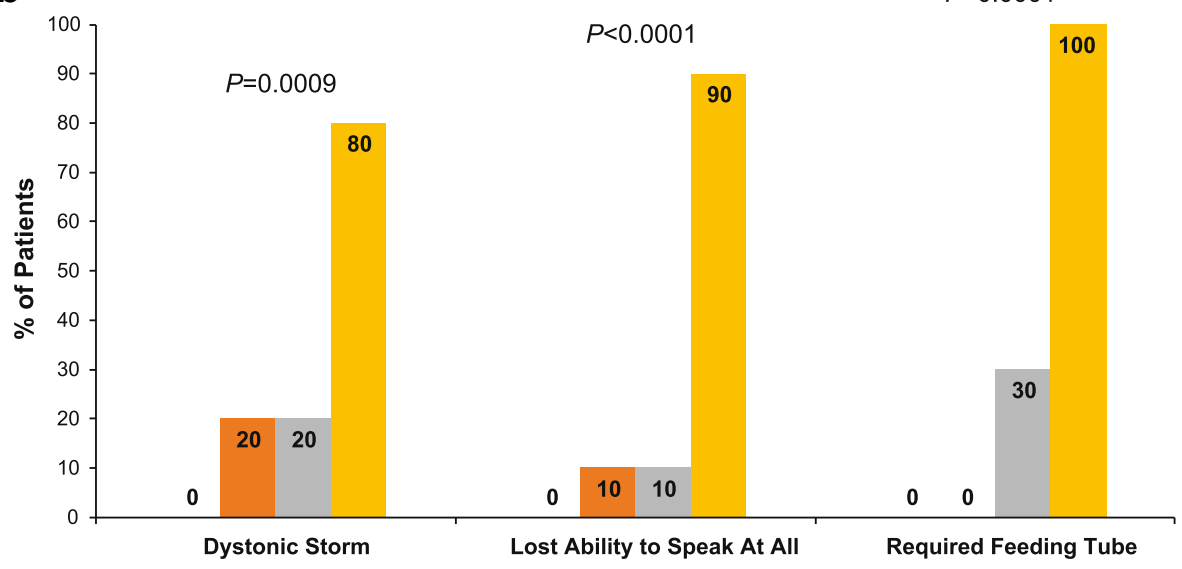

Lowest $(n=9) \quad$ Second Lowest $(n=10) \quad$ Third Lowest $(n=10) \quad$ Highest $(n=10)$

\section{C}

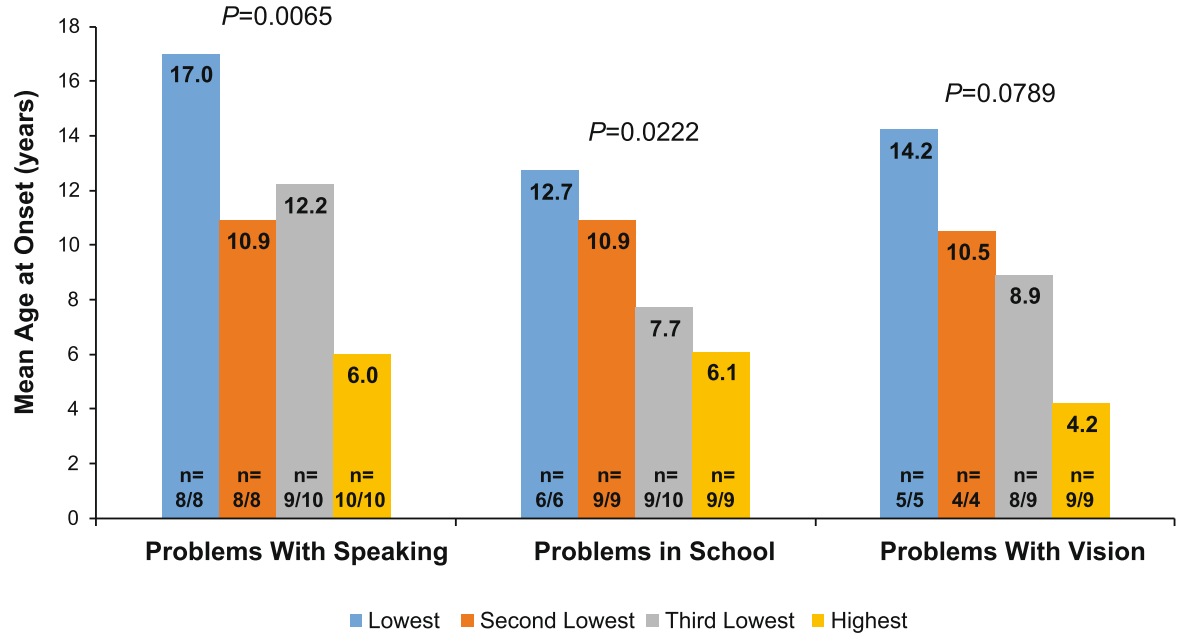

Fig. 2 PKAN symptoms and functional limitations with increased frequency across the spectrum of PKAN severity (a), Greater frequency in the Highest Severity group (b), or younger age at onset across PKAN severity (c). In panel $C$, the $n$ values show the actual number of patients reporting age at onset (numerator) and the total number of patients who experienced the problem (denominator) within each severity group. PKAN, pantothenate kinase-associated neurodegeneration 
Third Lowest severity group and the Highest severity group required the placement of a tracheostomy tube.

\section{Dystonic storms}

The percentages of patients who had ever experienced a dystonic storm were significantly different across PKAN severity groups $(P=0.0009)$, being more common in the Highest severity group (Fig. 2b). There was no significant difference in age at onset across PKAN severity groups $(P=0.3735)$, ranging from a mean (SD) age of 11.0 (5.7) years (Third Lowest group) to 20.0 (4.2) years (Second Lowest group).

\section{Problems in school of any kind related to PKAN}

Most patients $(34 / 39,87.2 \%)$ had problems in school. There was no significant difference in school problems across PKAN severity groups $(P=0.1717)$, ranging from $66.7 \%$ (6/9 Lowest group) to 100\% (10/10 Third Lowest group). Ages at onset for school problems were significantly different across PKAN severity groups $(P=0.0222)$, showing a decrease in mean age across the disease spectrum (Fig. 2c).

\section{Thematic analyses of burden of illness}

Thematic analyses of spontaneous statements during the patient and caregiver interviews were used to identify key areas of illness burden for patients. Caregivers mentioned patients' struggles to adapt to multiple losses of functioning (mobility, speech, ability to eat, visual impairment), loss of privacy due to the need for a caregiver, and social isolation because of loss of mobility, educational difficulties, difficulties communicating, and being moved out of their social group to special education programs. Examples of speech problems spontaneously reported by caregivers and patients include mumbling or words being garbled. Examples of reported school problems include the need for a modified program or for a full-time personal aide or nurse in the classroom, emotional and behavioral problems, and problems with handwriting or speech. Additionally, some caregivers spontaneously mentioned anger outbursts and mood swings in patients that they described as having difficulty with adjustment to the diagnosis. Painful episodes of dystonia and dystonic storms were described as adding to the patient burden.

\section{Discussion}

These data present a clinically rich perspective on patients with PKAN across a range of functional impairment. Because these data were collected from caregiver and patient interviews, rather than physician records, they offer a unique perspective in relation to other similar size case series. Moreover, the 35 US patients may have represented $6.0-12.0 \%$ of the estimated US population with PKAN (US prevalence estimated as $n=318-636$ [15]), and there was broad geographic representation.

There are several noteworthy findings. In general, severity of symptoms and problems, key medical history milestones, and burden of illness varied as would be expected with functional impairment, further supporting the validity of the PKAN-ADL [10]. Nearly $70.0 \%$ of families reported that the patient had problems with walking as a presenting symptom. This might include a range of neurological causes, including dystonia and parkinsonism. High-severity PKAN presented uniformly with walking difficulties, whereas heterogeneity of presentation for lower severity groups may contribute to delayed diagnosis. The median age of PKAN symptom onset of 7.0 years represented a wide range of ages $(<1.0$ 20.0 years). In nearly half the sample, the first symptom did not lead to a physician encounter, suggesting an insidious onset that only in retrospect is consistent with PKAN.

As with many rare diseases, a delay of up to several years prior to receiving an accurate diagnosis, over multiple physician visits, was the norm across the spectrum of PKAN severity. On average, the delay from symptom onset to diagnosis ranged from $\sim 2.5$ to 5.5 years, and the average delay from symptom onset to diagnosis based on genetic testing ranged from 3 to almost 6 years. Despite the "eye-of-the-tiger" sign on MRI being widely cited as near pathognomonic [8], only slightly more than half of the patients were diagnosed based on the first MRI. This finding suggests that the eye-of-the-tiger sign may not be as universally present as previously thought [16]. Alternatively, it could indicate the need for ongoing physician education to improve recognition of this radiological sign when it is present for PKAN diagnosis. It is also possible that for some of these patients, genetic testing may have confirmed the PKAN diagnosis prior to first MRI. Further study of the presenting features of PKAN in relation to disease severity and progression may inform earlier diagnosis and treatment.

These data reveal the extraordinary burden of illness borne by most families and patients across the spectrum of PKAN severity. Patients struggle with multiple losses as their functioning deteriorates through disease progression. Caregivers devote themselves part-time to full-time to assisting with daily activities, and transporting patients to a large number of medical visits, with an average of 13 physician visits and 55 therapy visits annually. Although economic status was not directly assessed, change in caregiver employment status implies a change in economic status. Finally, the combined costs of medical expenses (medical equipment and supplies, doctor's visits, surgeries, therapy visits), expenses for paid caregiving, and transportation expenses almost certainly create substantial economic burden for caregivers. 
Patients with PKAN across the spectrum of severity of functional impairment did not significantly differ in duration of illness (ranging from 9.7 years in the Second Lowest group to 15.2 years in the Third Lowest group), in contrast to the finding of Darling and colleagues (2017) [17] in a similarly sized cohort. This may reflect differences in the two cohorts, or in the scales themselves. Patients in the higher severity groups had younger age at onset for PKAN symptoms and functional impairments. The median age at symptom onset across the spectrum of PKAN severity ranged from 1.0 years old in the Highest severity group, to 7.0 and 10.0 years old in the Third Lowest and Second Lowest groups, respectively, to 14.0 years old in the Lowest group, but with broad overlapping ranges $(<1.0-12.0 ; 1.0-16.0$; 1.0-18.0; and 7.0-20.0, respectively), making age at onset itself of little practical use for disease subtyping. The most parsimonious explanation for these data is that PKAN is best viewed as a phenotypic spectrum rather than a disease with distinct subtypes ("classic" versus "atypical"), similar to many other inherited disorders.

A limitation of this study is its relatively small sample size, which may limit generalizability, although this is also a limitation with other case series in PKAN (eg, Sachin et al. 2009 [5] [ $n=16]$; Tomic et al. 2015 [7] $[n=9]$; Hayflick et al. 2003 [2] [ $n=123]$; and Hartig et al. $2006[1][n=72])$. This study's design did not include physician ratings or records. However, no other published study to date reports a detailed analysis of the patient journey, and patient and caregiver burden, in relation to overall functional impairment, which is the objective of this report. Our cohort was primarily drawn from North America and there may be geographic differences in mutation distribution [18]. We recruited mostly caregivers into the study rather than patients, which reflects the clinical reality that difficulty with communication and cognitive disability are core functional impairments of this disease, making verbal interviews with patients difficult or impossible.

\section{Conclusions}

These data derived from interviews with patients and caregivers help to further elucidate the diagnostic pathway and clinical experiences associated with PKAN. Across the spectrum of disease severity, PKAN is associated generally with a pre-adolescent age at onset, can be difficult to diagnose even with MRI, has a considerable burden of functional impairments, and has high healthcare utilization. Improved understanding of the real-world implications of PKAN across the spectrum of disease severity for patients and their caregivers can guide therapeutic planning and multi-functional medical team management.

\section{Additional file}

Additional file 1: Table S1. PKAN-specific medical history form. (DOCX $18 \mathrm{~kb})$

\section{Acknowledgements}

The authors would like to express their gratitude to Patricia Wood, Angelika Klucken, and Antonio Lopez, whose contributions to this project were essential to its success. Their courage as caregivers and leadership as patient advocates are examples to us all. Editorial and writing support was provided by Lynanne McGuire, PhD, CMPP of MedVal Scientific Information Services, LLC (Princeton, NJ) and was funded by Retrophin, Inc.

\section{Previous presentation}

Jinnah HA, et al. Presented at the American Neurological Association 2018 Annual Meeting, October 21-23, 2018, Atlanta, GA. Poster S224.

Jinnah HA, et al. Presented at the International Congress of Parkinson's Disease and Movement Disorders, October 5-9, 2018, Hong Kong, China. Poster 484.

Escolar ML, et al. Presented at the Child Neurology Society 2018 Annual Meeting, October 15-18, Chicago, IL. Poster 103.

\section{Authors' contributions}

RDM, AC, MLE, HAJ, TK, MCK, AV, ARV, LLS, DAR, RHB, and WRL conceived and designed the study. RDM, CB, LLS, DAR, RHB, and WRL designed and performed the statistical analysis, which was reviewed by all authors. All authors contributed to the first draft of the manuscript, reviewed and critically revised subsequent drafts, and gave final approval for submission.

\section{Funding}

Retrophin, Inc. provided the funding for the study. Evidera received funding from Retrophin, Inc. to participate in the study and for the development of the first draft of this manuscript. Employees of the study sponsor were involved in the design of the study; in the collection, analysis, and interpretation of data; and in writing the manuscript. This manuscript was prepared according to the International Society for Medical Publication Professionals' "Good Publication Practice for Communicating CompanySponsored Medical Research: GPP3."

\section{Availability of data and materials}

The data that support the findings of this study are available from the corresponding author on request.

\section{Ethics approval and consent to participate}

The study protocol received prior approval by Ethical and Independent Review Services, a central IRB. Interested potential participants were mailed a study packet that included two copies of the informed consent and assent forms. Evidera staff explained the study by telephone and obtained written and verbal informed consent for participants prior to the first data collection interview. Participants returned the signed informed consent forms in a postage-paid envelope.

\section{Consent for publication}

Not applicable.

\section{Competing interests}

RDM was formerly an employee of Retrophin, Inc. and may have an equity or other financial interest in Retrophin, Inc.

AC has provided consultant services to Ultragenyx, Inc. and Retrophin, Inc. MLE has provided consultant services to Retrophin, Inc.

HAJ has provided consultant services to Retrophin, Inc. and has active or recent grant support from the US government (National Institutes of Health), private philanthropic organizations (the Benign Essential Blepharospasm Research Foundation, Cure Dystonia Now), academically-oriented institutions (the Dystonia Study Group), and industry (Cavion Therapeutics, Ipsen Pharmaceuticals, Retrophin Inc.); has served on advisory boards or as a consultant for Abide Therapeutics, Allergan Inc., Psyadon Pharmaceuticals, Saol Therapeutics, and Medtronic Inc.; has received honoraria or stipends for lectures or administrative work from the American Academy of Neurology, the Dystonia Medical Research Foundation, the International Neurotoxin 
Society, the International Parkinson's Disease and Movement Disorders Society, The Parkinson's Disease Foundation, and Tyler's Hope for a Cure; serves on the Scientific Advisory Boards for Cure Dystonia Now, the Dystonia Medical Research Foundation, Lesch-Nyhan Action France, and Tyler's Hope for a Cure; and is principle investigator for the Dystonia Coalition, which receives the majority of its support through NIH grant TR001456 from the Office of Rare Diseases Research at the National Center for Advancing Translational Sciences, and previously NS065701 from the National Institutes of Neurological Disorders and Stroke. The Dystonia Coalition has received additional material or administrative support from industry sponsors (Allergan Inc. and Merz Pharmaceuticals) as well as private foundations (The American Dystonia Society, Beat Dystonia, The Benign Essential Blepharospasm Foundation, Cure Dystonia Now, Dystonia Inc., Dystonia Ireland, The Dystonia Medical Research Foundation, The European Dystonia Federation, The Foundation for Dystonia Research, The National Spasmodic Dysphonia Association, and The National Spasmodic Torticollis Association).

TK did not receive any payment or honoraria from Retrophin, Inc. when the research was conducted, but is currently the coordinating investigator of a clinical study of fosmetpantotenate in PKAN, which is sponsored by Retrophin, Inc. He acknowledges support by the European Commission 7th Framework Programme (FP7/2007-2013, HEALTH-F2-2011, grant agreement No. 277984, TIRCON) and by the European Reference Network for Rare Neurological Diseases (ERN-RND) co-funded by the European Commission (ERN-RND: 3HP 767231). He is a coordinating investigator of the deferiprone in PKAN randomized and extension trial and received research funding from ApoPharma Inc. He provides consulting services to CoA Therapeutics and TM3 Therapeutics. He has received travel support from ApoPharma Inc. MCK has received grant support from Retrophin, Inc. and receives funding from both federal sources (US NIH NS106298) and foundation sources (Scott Foundation, CureCP, Cerebral Palsy Alliance Research Foundation). He serves on the Scientific Advisory Board of the Spastic Paraplegia Foundation and has served as a speaker for the Tourette Association of America and as a consultant for PTC Therapeutics.

AV has provided consultant services to Retrophin, Inc.

ARV has received grant funding from $\mathrm{NIH}$ and the McDonnell Center for Systems Neuroscience.

CB is an employee of Retrophin, Inc. and may have an equity or other financial interest in Retrophin, Inc.

LLS, DAR, and WRL are employees of Evidera, and were hired by Retrophin, Inc. to conduct the research. RHB was an employee of Evidera when the research was conducted. They did not receive any payment or honoraria directly from Retrophin, Inc. for services rendered. Evidera received funding from Retrophin, Inc. to participate in the study and the development of the first draft of this manuscript.

\section{Author details}

${ }^{1}$ Formerly Retrophin, Inc., San Diego, CA, USA. ${ }^{2}$ Departments of Pediatrics and Neurology, University of Colorado, School of Medicine, Denver, CO, USA. ${ }^{3}$ Department of Pediatrics, University of Pittsburgh School of Medicine, Pittsburgh, PA, USA. ${ }^{4}$ Departments of Neurology and Human Genetics, Emory University School of Medicine, Atlanta, GA, USA. ${ }^{5}$ Department of Neurology, Friedrich-Baur-Institute, University of Munich, Munich, Germany. ${ }^{6}$ German Center for Neurodegenerative Diseases (DZNE), Munich, Germany. ${ }^{7}$ Munich Cluster for Systems Neurology (SyNergy), Munich, Germany. ${ }^{8}$ Barrow Neurological Institute, Phoenix Children's Hospital, University of Arizona College of Medicine, Phoenix, AZ, USA. 'Department of Neurology, Massachusetts General Hospital/Harvard Medical School, Boston, MA, USA. ${ }^{10}$ Department of Neurology, Washington University School of Medicine, St. Louis, MO, USA. ${ }^{11}$ Retrophin, Inc., 3721 Valley Centre Drive, Suite 200, San Diego, CA 92130, USA. ${ }^{12}$ Evidera, Inc, Bethesda, MD, USA.

Received: 1 March 2019 Accepted: 25 June 2019

Published online: 12 July 2019

\section{References}

1. Hartig MB, Hortnagel K, Garavaglia B, et al. Genotypic and phenotypic spectrum of PANK2 mutations in patients with neurodegeneration with brain iron accumulation. Ann Neurol. 2006;59(2):248-56.

2. Hayflick SJ, Westaway SK, Levinson B, et al. Genetic, clinical, and radiographic delineation of Hallervorden-Spatz syndrome. N Engl J Med. 2003;348(1):33-40.
3. Lee JH, Park J, Ryu HS, et al. Clinical heterogeneity of atypical pantothenate kinase-associated neurodegeneration in Koreans. J Mov Disord. 2016;9(1):20-7.

4. National Organization for Rare Disorders. Pantothenate kinase-associated neurodegeneration. 2016; Available at: https://rarediseases.org/rarediseases/pantothenate-kinase-associated-neurodegeneration/. Accessed 27 Nov 2017

5. Sachin S, Goyal V, Singh S, et al. Clinical spectrum of Hallervorden-Spatz syndrome in India. J Clin Neurosci. 2009;16(2):253-8.

6. Stamelou M, Lai SC, Aggarwal A, et al. Dystonic opisthotonus: a "red flag" for neurodegeneration with brain iron accumulation syndromes? Mov Disord. 2013;28(10):1325-9.

7. Tomić A, Petrović I, Svetel M, Dobričić V, Dragašević Mišković N, Kostic VS. Pattern of disease progression in atypical form of pantothenate-kinaseassociated neurodegeneration (PKAN) - prospective study. Parkinsonism Relat Disord. 2015;21(5):521-4.

8. Hogarth P, Kurian MA, Gregory A, et al. Consensus clinical management guideline for pantothenate kinase-associated neurodegeneration (PKAN). Mol Genet Metab. 2017;120(3):278-87.

9. Marelli C, Piacentini S, Garavaglia B, Girotti F, Albanese A. Clinical and neuropsychological correlates in two brothers with pantothenate kinaseassociated neurodegeneration. Mov Disord. 2005;20(2):208-12.

10. Marshall RD, Collins A, Escolar ML, et al. A scale to assess activities of daily living in pantothenate kinase-associated neurodegeneration. Mov Disord Clin Pract. 2019;6(2):139-49.

11. Fahn S, Elton R. Recent developments in Parkinson's disease, vol. 2. Florham Park: Macmillan Health Care Information; 1987.

12. Furlong W, Feeny D, Torrance G. 2016 health utilities index ${ }^{\circledast}$ mark 2 and mark 3 (HUI2/3) 40-item questionnaire for interviewer-administered, proxyassessed "usual" health status assessment. Dundas: Health Utilities, Inc.; 2016.

13. Sutcliffe LM, Lincoln NB. The assessment of depression in aphasic stroke patients: the development of the stroke aphasic depression questionnaire. Clin Rehabil. 1998;12(6):506-13.

14. Riessman C. Narrative methods for the human sciences. Thousand Oaks: SAGE Publications; 2008

15. Orphanet. Pantothenate kinase-associated neurodegeneration. 2018; Available at: http://www.orpha.net/consor/cgi-bin/OC_Exp.php?Expert= 157850. Accessed 25 Jan 2019.

16. Kruer M. Pantothenate kinase-associated neurodegeneration. In: Rosenberg R, Pascual J, editors. Rosenberg's molecular and genetic basis of neurological and psychiatric disease. 5th ed. London: Academic Press; 2015. p. 473-81.

17. Darling A, Tello C, Marti MJ, et al. Clinical rating scale for pantothenate kinase-associated neurodegeneration: a pilot study. Mov Disord. 2017; 32(11):1620-30.

18. Delgado RF, Sanchez PR, Speckter H, et al. Missense PANK2 mutation without "eye of the tiger" sign: MR findings in a large group of patients with pantothenate kinase-associated neurodegeneration (PKAN). J Magn Reson Imaging. 2012;35(4):788-94.

\section{Publisher's Note}

Springer Nature remains neutral with regard to jurisdictional claims in published maps and institutional affiliations.

Ready to submit your research? Choose BMC and benefit from:

- fast, convenient online submission

- thorough peer review by experienced researchers in your field

- rapid publication on acceptance

- support for research data, including large and complex data types

- gold Open Access which fosters wider collaboration and increased citations

- maximum visibility for your research: over $100 \mathrm{M}$ website views per year

At $\mathrm{BMC}$, research is always in progress.

Learn more biomedcentral.com/submissions 